\title{
The Mechanism of the Anodic Oxidation of Hexamethylbenzene to the Pentamethylbenzyl Cation in Dichloromethane
}

\author{
JIRI BAREK,* ELISABET AHLBERG and VERNON D. PARKER **
}

Organic Chemistry Laboratories, Norwegian Institute of Technology, University of Trondheim, N-7034 Trondheim-NTH, Norway

The deprotonation of the hexamethylbenzene cation radical in dichloromethane containing trifluoroacetic acid was studied using cyclic voltammetry and double step chronoampermetry as diagnostic techniques. The initial deprotonation was found to be reversible, causing the overall rate of the reaction to be inversely proportional to the acid concentration. Furthermore, the reaction was found to be second order in the cation radical which implicates the homogeneous electron transfer (iii) as one of the rate determining steps. A deuterium kinetic isotope effect of $2 \pm 0.5$ was found during studies on the perdeuterated substrate. The kinetic data are consistent with the following complex mechanism.

$$
\begin{aligned}
& \mathrm{R}-\mathrm{H}-\mathrm{e}^{-} \rightleftarrows(\mathrm{R}-\mathrm{H})^{+} \\
& (\mathrm{R}-\mathrm{H})^{+} \rightleftarrows \mathrm{R}+\mathrm{H}^{+} \\
& \mathrm{R}+(\mathrm{R}-\mathrm{H})^{+} \rightarrow \mathrm{R}^{+}+\mathrm{RH}
\end{aligned}
$$

The reactions following the one electron oxidation of alkylbenzenes and related compounds have received a great deal of attention. Eberson ${ }^{1}$ has recently discussed the mechanism in detail and related the energetics of the initial step, the deprotonation assisted by a base, to the corresponding reaction between the hydrocarbon and a free radical. A common intermediate, the benzyl radical, is involved in both of these reactions. The discussion referred to above ${ }^{1}$ as well as a number of others ${ }^{2-5}$ concentrates on the case where the deprotonation is the rate determining step in the disappearance of

* On leave from Department of Analytical Chemistry, Charles University, Prague, Czechoslovakia.

** To whom correspondence should be addressed. the cation radical and where this step is irreversible. The mechanism under these conditions is simply the reaction illustrated in eqn. (1).

$\left(\mathrm{Ar}-\mathrm{CH}_{3}\right)^{++}+\mathrm{B}^{-} \rightarrow \mathrm{Ar}-\mathrm{CH}_{2}+\mathrm{BH}$

During kinetic studies on the reactions of the hexamethylbenzene cation radical (HMB) we have now found that the reaction in dichloromethane is much more complicated than indicated by (1) and that the homogeneous electron transfer between the cation radical and the intermediate benzyl radical contributes to the determination of the overall rate of the reaction.

\section{RESULTS}

Attempts to observe the hexamethylbenzene cation radical either by cyclic voltammetry or by double step chronoamperometry ${ }^{6}$ in acetonitrile failed at temperatures as low as $-40{ }^{\circ} \mathrm{C}$. This indicates that the lifetime of the one electron oxidation product of HMB is less than about $10^{-4} \mathrm{~s}$. In dichloromethane similar results were obtained but the addition of trifluoroacetic acid (TFA) to the voltammetric solution resulted in significant enhancement of the lifetime so that nearly reversible cyclic voltammograms could be obtained at high voltage sweep rates.

Double Potential Step Chronoamperometric Studies. The method of data analysis was similar to that published by Bard. ${ }^{7}$ Current time curves were obtained by stepping the potential about $200 \mathrm{mV}$ more positive than the peak potential for the oxidation of HMB and back to the starting potential, 
about $200 \mathrm{mV}$ less positive than the foot of the oxidation wave. The current was measured at $t_{\mathrm{f}}$ (time just before the second potential step) and at $t_{\mathrm{b}}\left(=2 t_{\mathrm{f}}\right)$. The current ratio $I_{\mathrm{b}} / I_{\mathrm{f}}$ was normalized for loss of cation radical by diffusion by dividing by $\left(1-2^{-\frac{1}{2}}\right)$ in accordance with the calculations of Schwartz and Shain. ${ }^{6}$ Plots of the normalized current ratios $\left(R_{\mathrm{I}}\right)$ obtained from potential steps varying widely in $t_{\mathrm{f}}$ were used to obtain $t_{\frac{1}{2}}$, defined as the value of $t_{\mathrm{f}}$ where $R_{\mathrm{I}}$ is equal to 0.5 . Finally, $R_{\mathrm{I}}$ was plotted $v s$. time in units of $t_{\frac{1}{2}}$ and the resulting curves were compared with those calculated using digital simulation. $^{8}$

First order mechanism. The formation of $\mathrm{HMB}^{+}$ at the anode followed by reaction (1) and subsequently a fast homogeneous electron transfer to generate the benzyl cation is a form of the ECE mechanism, eqns. (2) - (4). The chronoamperometric working curve for this mechanism is shown in

$$
\begin{aligned}
& \mathrm{A}-\mathrm{e} \rightleftarrows \mathrm{A}^{++} \\
& \mathrm{A}^{++} \rightarrow \mathrm{B} \\
& \mathrm{B}+\mathrm{A}^{\cdot+} \rightleftarrows \mathrm{C}+\mathrm{A}
\end{aligned}
$$

Fig. 1 along with the data for the oxidation of HMB in dichloromethane containing TFA (5.26 M). The experimental data at long times deviate significantly from the theoretical curve suggesting that the reaction does not follow the ECE mechanism. This was further demonstrated by changing

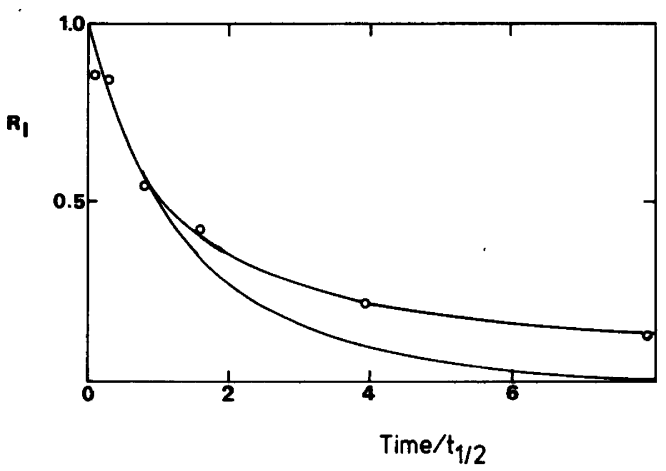

Fig. 1. Double step chronoamperometric data (circles) for the oxidation of HMB in dichloromethane - TFA $(40 \%)$ containing $\mathrm{Bu}_{4} \mathrm{NBF}_{4}(0.2 \mathrm{M})$ and working curves of second order mechanism (solid line, upper curve) and the ECE mechanism (solid line, lower curve). the substrate concentration. Rate constants calculated on the basis of the ECE working curve increased dramatically with increasing concentration.

Second order mechanism. A second order mechanism for the formation of the benzyl cation from $\mathrm{HMB}^{+}$would have to involve the homogeneous electron transfer in the rate determining step. Another factor which must be taken into account is that the rate of the reaction is strongly dependent upon the TFA concentration as was demonstrated by the cyclic voltammetric study. This implicates a reversible deprotonation-protonation equilibrium. Working curves were calculated for the mechanism illustrated in (5) $-(7)$, assuming that equilibrium

$\mathrm{A}-\mathrm{e} \rightleftarrows \mathrm{AH}^{++}$

$\mathrm{AH}^{++} \rightleftarrows \mathrm{A}^{+}+\mathrm{H}^{+}$

$\mathrm{A}^{-}+\mathrm{AH}^{++} \rightleftarrows \mathrm{A}^{+}+\mathrm{AH}$

(6) is fast and the rate is determined by (7). The working curve calculated for this mechanism gives an apparent rate constant, dependent upon the rate constant for reaction (7), the equilibrium constant for (6) and the concentration of the proton

Table 1. Calculated double potential step chronoamperometric data for the deprotonation equilibrium followed by rate determining homogeneous electron transfer.

\begin{tabular}{ll}
\hline$R_{\mathrm{I}}{ }^{a}$ & Time $/ t_{\frac{1}{2}}{ }^{b}$ \\
\hline 1.0 & 0 \\
0.853 & 0.177 \\
0.793 & 0.265 \\
0.741 & 0.354 \\
0.587 & 0.708 \\
0.487 & 1.06 \\
0.416 & 1.42 \\
0.364 & 1.77 \\
0.323 & 2.12 \\
0.292 & 2.48 \\
0.265 & 2.83 \\
0.244 & 3.18 \\
0.196 & 4.25 \\
0.157 & 5.66 \\
0.131 & 7.08 \\
\hline
\end{tabular}

${ }^{a}$ The ratio of $I_{\mathrm{b}} / I_{\mathrm{f}}$ (normalized). ${ }^{b}$ The unit of time is $t_{1}$, the magnitude of which is dependent upon the kinetic parameters. 


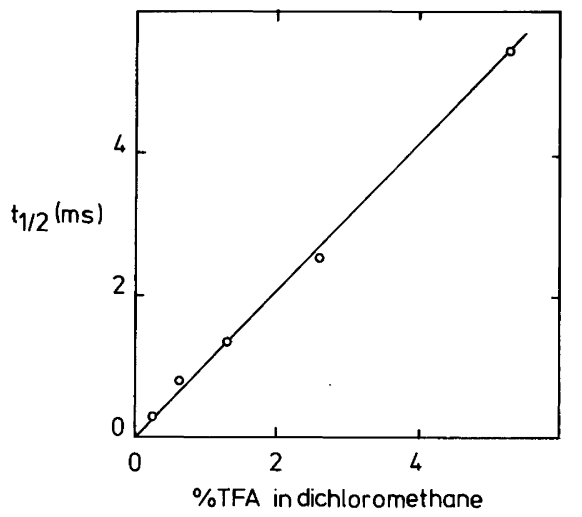

Fig. 2. Dependence of the chronoamperometric half-time for the oxidation of HMB in dichloromethane upon the TFA concentration.

donor as shown in (8) where $C_{\mathrm{HMB}}^{\mathrm{b}}$ is the bulk concentration. The apparent rate constant, $k_{\text {app }}$,

$k_{\mathrm{app}}=k_{7} K_{6} /\left(\mathrm{H}^{+}\right)=0.565 / C_{\mathrm{HMB}}^{\mathrm{b}} t_{\frac{1}{2}}$ can be obtained directly from $t_{\frac{1}{2}}$. The data obtained by digital simulation ${ }^{8}$ is summarized in Table 1 and the working curve along with the experimental data for the oxidation of HMB in dichloromethane containing TFA $(5.26 \mathrm{M})$ are illustrated by the upper curve in Fig. 1.

Effect of the TFA concentration on the rate of deprotonation. The chronoamperometric half-time for the oxidation of HMB in dichloromethane as a function of (TFA) is illustrated in Fig. 2. A reasonably good linear correlation is observed with $t_{\frac{1}{2}}$ approaching an immeasurably small value as the acid concentration approaches zero. The values of $k_{\text {app }}$ calculated according to (8) are summarized in Table 2. The last column in Table 2 shows that $k_{\text {app }}$ normalized for (TFA) is very nearly constant for this set of experiments where the concentration of $\mathrm{HMB}$ was held at $10 \mathrm{mM}$.

Effect on $H M B$ concentration on the rate of formation of the pentamethylbenzyl cation. A set of experiments was carried out in which the concentration of TFA was held constant at $5.26 \mathrm{M}$ and the HMB concentration was varied from 1 to $10 \mathrm{mM}$. A plot of $1 / t_{\frac{1}{2}}$ as a function of (HMB) is

Table 2. Effect of TFA concentration on the kinetics of the deprotonation of hexamethylbenzene cation radical in dichloromethane at $20^{\circ} \mathrm{C} .^{a}$

\begin{tabular}{llll}
\hline [TFA] & $t_{\frac{1}{2}} / \mathrm{ms}^{b}$ & $\left(10^{-4}\right) k_{\mathrm{app}} /(\mathrm{Ms})^{-1 \mathrm{c}}$ & $\left(10^{-4}\right) k_{\mathrm{app}}(\mathrm{TFA}) / \mathrm{s}^{-1 \mathrm{c}}$ \\
\hline 0.26 & 0.3 & 18.8 & 4.88 \\
0.66 & 0.8 & 7.06 & 4.66 \\
1.32 & 1.35 & 4.19 & 5.53 \\
2.63 & 2.55 & 2.22 & 5.84 \\
5.26 & 5.5 & 1.03 & 5.42 \\
\hline
\end{tabular}

${ }^{a}$ Supporting electrolyte $=\mathrm{Bu}_{4} \mathrm{NBF}_{4}(0.2 \mathrm{M}),[\mathrm{HMB}]=10 \mathrm{mM} .{ }^{b}$ Chronoamperometric; half-time as defined in the text. ${ }^{c}$ Calculated according to eqn. (8).

Table 3. Effect of hexamethylbenzene concentration on the kinetics of the deprotonation of hexamethylbenzene cation radical in dichloromethane at $20^{\circ} \mathrm{C} .^{a}$

\begin{tabular}{llll}
\hline$[\mathrm{HMB}] / \mathrm{mM}$ & $t_{\frac{1}{2}} / \mathrm{ms}^{b}$ & $\left(10^{-4}\right) k_{\mathrm{app}} /(\mathrm{Ms})^{-1 c}$ & $\left(10^{-4}\right) k_{\mathrm{app}}[\mathrm{TFA}] / \mathrm{s}^{-1 c}$ \\
\hline 1.0 & 40 & 1.41 & 7.42 \\
2.0 & 20 & 1.41 & 7.42 \\
4.0 & 12 & 1.18 & 6.21 \\
6.0 & 9 & 1.05 & 5.52 \\
8.0 & 7 & 1.01 & 5.31 \\
10.0 & 5.5 & 1.03 & 5.42 \\
\hline
\end{tabular}

\footnotetext{
${ }^{a}$ Supporting electrolyte $=\mathrm{Bu}_{4} \mathrm{NBF}_{4}(0.2 \mathrm{M}),[\mathrm{TFA}]=5.26 \mathrm{M} \cdot{ }^{b}$ Chronoamperometric; half-time as defined in the text. ${ }^{c}$ Calculated according to eqn. (8).
}

Acta Chem. Scand. B 34 (1980) No. 2 


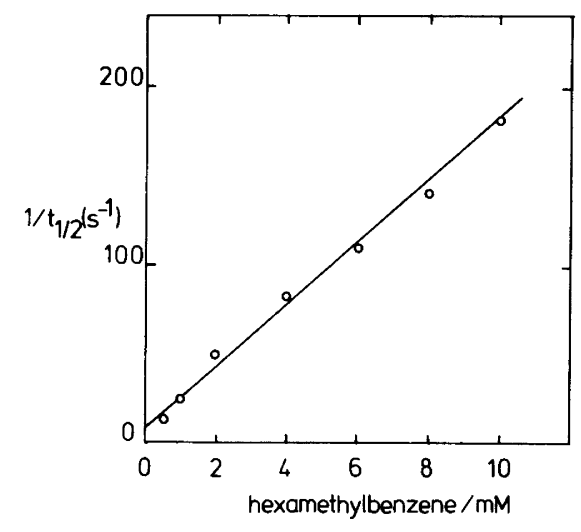

Fig. 3. Dependence of the reciprocal of the chronoamperometric half-time on the HMB concentration during oxidation in dichloromethane-TFA $(40 \%)$.

shown in Fig. 3. Once again a good linear correlation is observed. The data are summarized in Table 3 which shows that $k_{\text {app }}$ is very nearly constant at an acid concentration of $5.26 \mathrm{M}$. The last column is $k_{\text {app }}$ multiplied by the TFA concentration for comparison with the data in Table 2.

Deuterium kinetic isotope effect. A kinetic study of the reaction of the cation radical of the perdeuterio compound $\mathrm{C}_{12} \mathrm{D}_{18}$ in dichloromethane containing $\mathrm{Bu}_{4} \mathrm{NBF}_{4}(0.2 \mathrm{M})$ and TFA $(5.26 \mathrm{M})$ resulted in a value of $k_{\text {app }}$ equal to $6.4( \pm 0.4) \times 10^{3}$ $\mathrm{M}^{-1} \mathrm{~s}^{-1}$ indicating a deuterium kinetic isotope effect of $2 \pm 0.5$.

\section{DISCUSSION}

Since the deprotonation of a methylarene cation radical is a simple acid-base reaction (9), the position of the equilibrium as well as the rates of reaction in both directions should be very dependent upon the nature of the medium and the presence of bases. The solvent system, dichloromethane-TFA, is a strongly acidic medium and the kinetic results

$$
\begin{aligned}
& \left(\mathrm{Ar}-\mathrm{CH}_{3}\right)^{++}+\mathrm{B} \underset{k_{-9}}{\stackrel{k_{9}}{\rightleftarrows}} \mathrm{Ar}-\mathrm{CH}_{2}+\mathrm{BH}^{+} \\
& \mathrm{Ar}-\mathrm{CH}_{2}+\left(\mathrm{Ar}-\mathrm{CH}_{3}\right)^{+} \underset{k_{-10}}{\stackrel{k_{10}}{\rightleftarrows}} \mathrm{Ar}-\mathrm{CH}_{2}^{+}+\mathrm{ArCH}_{3}
\end{aligned}
$$

$$
\mathrm{Ar}-\mathrm{CH}_{2}^{+}+\text {nucleophile } \stackrel{k_{11}}{\longrightarrow} \text { products }
$$

with (HMB) ${ }^{+}$indicate that equilibrium (9) lies to the left in this case. If we assume that equilibrium (10) is exothermic as one would predict from electrode potentials measured in related systems, ${ }^{9}$ we can estimate the magnitudes of the equilibrium constants and rate constants in (9). If (10) is exothermic with $\Delta G^{\circ}$ equal to or greater than about $-0.2 \mathrm{eV}, k_{10}$ is predicted to be a diffusion controlled rate constant ${ }^{10}$ equal to approximately $10^{10} \mathrm{M}^{-1}$ $\mathrm{s}^{-1}$. Eqn. (12) would then describe the relationship between the apparent rate constant and the equilibrium constant for reaction (9). The product of

$[\mathrm{TFA}] k_{\mathrm{app}}=k_{10} K_{9}=\left(10^{10}\right) K_{9}$

$k_{\text {app }}$ and [TFA] observed in this study (Tables 2 and 3), was of the order of $5 \times 10^{4}$ which leads to a value of $2.6 \times 10^{-5}$ for $K_{9}$ at a TFA concentration of $5.26 \mathrm{M}$. Making the further reasonable assumption that $k_{-9}$, the rate constant for the exothermic proton transfer, is a diffusion controlled rate constant allows us to make an estimate of the value of $k_{9}$ at $2.6 \times 10^{5} \mathrm{M}^{-1} \mathrm{~s}^{-1}$. The approximate nature of such an estimate must be emphasized since in this case the nature of $B$ in (9) is not known and is not taken into account in the simulation of the working curve which was used to determine $k_{\text {app }}$. In going to acetonitrile as solvent, a much more basic medium, one would predict that equilibrium (9) should lie further to the right by virtue of the increased concentration of B which would result in an increased value of $k_{\text {app }}$. This is, in fact, what is observed. The overall reaction in acetonitrile was found to be too rapid to measure the rates by double step chronoamperometry even at $-40{ }^{\circ} \mathrm{C}$ and [TFA] in excess of $1 \mathrm{M}$.

The kinetics of the formation of benzyl radicals during pulse radiolysis of aqueous acid solutions of methylarenes, which involves the intermediate formation of the corresponding cation radicals, have been reported. ${ }^{11}$ Pseudo first order rate constants ranging from $10^{7} \mathrm{~s}^{-1}$ for toluene down to $10^{4} \mathrm{~s}^{-1}$ for pentamethylbenzene were observed in aqueous solutions. Unfortunately, HMB was not included in the latter study. Eberson ${ }^{1}$ estimated that the rate constant for the deprotonation of $(\mathrm{HMB})^{\cdot+}$ should be of the same order of magnitude as that for the cation radical of pentamethylbenzene which leads to a value of about $10^{4} \mathrm{~s}^{-1}$ for the pseudo first order rate constant in water. The deprotonation reaction during the pulse radiolysis study was considered as the simple irreversible reaction (1). 
However, the rate of deprotonation of the cation radicals was found to be retarded by the presence of $\mathrm{HClO}_{4}$ in concentrations of $1 \mathrm{M}$ or greater. Attempts to detect the reverse reaction were not successful. The latter indicates that in aqueous solution equilibrium (9) lies to the right in contrast to our observations in dichloromethane-TFA. This is not surprising since acids in general are dissociated to a greater extent in water than in less polar media.

It is somewhat surprising that the deprotonation reactions of $(\mathrm{HMB})^{++}$in non-aqueous solvents are much faster than predicted from the results in aqueous solution. For example, the rate constant in acetonitrile, if it is a pseudo first order reaction, can be estimated to be $10^{5} \mathrm{~s}^{-1}$ or greater at room temperature based on our failure to observe the cation radical, while in water the rate constant is estimated to be about $10^{2} \mathrm{~s}^{-1}{ }^{1}$

In conclusion, it would be mentioned that the product forming reaction (11) under the conditions of our study has been observed to yield the corresponding trifluoroacetic acid ester in high yield. ${ }^{3,12}$

\section{EXPERIMENTAL}

Reagents, electrodes and cells. Reagent grade hexamethylbenzene was used without further purification. Dichloromethane containing the supporting electrolyte $\mathrm{Bu}_{4} \mathrm{NBF}_{4}(0.2 \mathrm{M})$ was passed through a column of neutral alumina before use. Tetrabutylammonium fluoroborate was prepared by ion exchange from the hydrogen sulfate (Hässle, Gothenburg) and aqueous fluoroboric acid, extracted into dichloromethane, precipitated with ether and dried. The working electrodes and reference electrodes were constructed in the manner previously described. ${ }^{13}$

Instrumentation. The potentiostat was a PAR model 173D with a PAR 176 current follower driven by a PPR1 waveform generator (HiTek Instruments, England). An AA1 multi-purpose signal averager (Hi-Tek) and a $7046 \mathrm{~A} X-Y Y$ recorder (Hewlett-Packard) were used to record the voltammetric data. Positive feed-back for $I R$ correction was adjusted until the onset of oscillation and then backed off slightly as recommended by Whitson. ${ }^{14}$ Data were collected at a rate of 5 $\mu \mathrm{s} /$ point and the precision of the signal averager is 10 bits.

Acknowledgements. J. B. was supported under the cultural agreement between Czechoslovakia and Norway and E. A. by the Norwegian Research Council for Science and the Humanities. We gratefully acknowledge this support. We thank Professor Enrico Baciocchi for a sample of perdeuteriohexamethylbenzene.

\section{REFERENCES}

1. Eberson, L., Jönsson, L. and Wistrand, L.-G. Acta Chem. Scand. B 32 (1978) 520.

2. Bewick, A., Edwards, G. J., Mellor, J. M. and Pons, S. J. Chem. Soc. Perkin. Trans. 2 (1977) 1952.

3. Svanholm, U. and Parker, V. D. Tetrahedron Lett. (1972) 471.

4. Kochi, J. K., Tang, R. T. and Bernath, T. J. Am. Chem. Soc. 95 (1973) 7114.

5. Heiba, E. I., Dessau, R. M. and Koehl, W. J., Jr. J. Am. Chem. Soc. 91 (1969) 6830.

6. Schwarz, W. M. and Shain, I. J. Phys. Chem. 69 (1965) 30.

7. Childs, W. V., Maloy, J. T., Keszthelyi, C. P. and Bard, A. J. J. Electrochem. Soc. 118 (1971) 874.

8. Feldberg, S. Electroanal. Chem. 3 (1969) 199.

9. Breslow, R. and Chu, W. J. Am. Chem. Soc. 95 (1973) 411.

10. Dorfman, L. M. Acc. Chem. Res. 3 (1970) 224.

11. Holcman, J. Thesis, Risø National Laboratory, DK-4000 Roskilde, Denmark.

12. Nyberg, K. and Trojanek, A. Collect. Czech. Chem. Commun. 40 (1975) 526.

13. Lines, R. and Parker, V. D. Acta Chem. Scand. B 31 (1977) 369.

14. Whitson, P. E., Vanden Born, H. W. and Evans, D. H. Anal. Chem. 45 (1973) 1298.

Received July 19, 1979. 